\title{
Omnidirectional Ultrasonic Localization for Mobile Robots
}

\author{
Chen-Chien Hsu, ${ }^{1}$ Hsin-Chuan Chen, ${ }^{2 *}$ Ching-Chang Wong, ${ }^{3}$ and Chien-Yu Lai ${ }^{4}$ \\ ${ }^{1}$ Department of Electrical Engineering, National Taiwan Normal University, Taipei City 10610, Taiwan \\ ${ }^{2}$ School of Computer Engineering, University of Electronic Science and Technology of China, \\ Zhongshan Institute, Zhongshan 528402, China \\ ${ }^{3}$ Department of Electrical and Computer Engineering, Tamkang University, New Taipei City 25137, Taiwan \\ ${ }^{4}$ Department of Electrical and Computer Engineering, Tamkang University, New Taipei City 25137, Taiwan
}

(Received April 28, 2021; accepted September 6, 2021; online published September 16, 2021)

Keywords: ultrasonic, omnidirectional sensing, localization, mobile robot, dual-circle localization

To solve the detection angle limitation of conventional ultrasonic sensors, we propose a localization system based on omnidirectional ultrasonic sensing by using a reflection cone to generate ultrasonic beams with $360^{\circ}$ propagation. The architecture of the proposed localization system includes four ultrasonic receivers deployed around a square measuring environment and a mobile robot carrying an integrated omnidirectional ultrasonic module as a transmitter. When the ultrasonic receivers receive ultrasonic signals from the integrated omnidirectional ultrasonic module, distance measurements between the transmitter and the receiver can be obtained by time-of-flight calculation, which are sequentially transferred to a remote PC via a Zigbee wireless network. Using the distance measurements collected from the receivers, the coordinates of the mobile robot can be determined by using the established dual-circle localization method. Experimental results have shown satisfactory accuracy in determining the coordinates of the mobile robot via the proposed localization system.

\section{Introduction}

It is critically important for a mobile robot to move from place to place without running into obstacles or getting lost. The successful management of this navigation task depends on a robust and reliable solution to the estimation of the robot position in the world space. ${ }^{(1)}$ The current trend toward the self-localization of mobile robots in an indoor environment has been extensively studied $^{(2-4)}$ and has many practical industrial applications, for example, real-time moving object monitoring. Over the past years, many methods by which mobile robots can avoid obstacles have been developed. Multisensor information fusion and a control algorithm based on a fuzzy neural network $^{(5)}$ were proposed for obstacle avoidance by mobile robots. Moreover, some researchers deployed an OmniHyper camera on a robot to capture $360^{\circ}$ circular images for image processing, so that the robot can successfully avoid obstacles by combining an improved dynamic window approach with an artificial potential field for path planning. ${ }^{(6)}$

Among the localization methods, the Global Positioning System (GPS) ${ }^{(7)}$ is regarded as a popular and versatile approach that has been used in a wide range of applications. ${ }^{(8,9)}$

*Corresponding author: e-mail: chen_robin@,foxmail.com

https://doi.org/10.18494/SAM3419 
Unfortunately, signals are seriously blocked when GPS is used indoors. As a result, GPS is not suitable for indoor environments in general. Various indoor localization techniques have therefore been developed in recent years using different sensors, including approaches based on lasers, vision, RFID, Zigbee, ultrasonics, and wireless networks. Although laser-based approaches are accurate for localization, ${ }^{(10,11)}$ the cost of implementation is very high for lowcost applications. Although vision-based approaches ${ }^{(12,13)}$ provide a rich source of environment information, they may require intensive computation and be time-consuming. One of the applications of a stereo vision-based self-localization system ${ }^{(14)}$ was in the 2010 RoboCup soccer humanoid league competition, where it was used to increase the precision of distance measurement by an artificial neural network technique. Signal-strength-based localization techniques, including RFID, ${ }^{(15)}$ Zigbee, ${ }^{(16)}$ and wireless networks, ${ }^{(17)}$ however, suffer from inaccurate measurements because the signal strength is generally deteriorated by various noises in the environment. The other indoor localization approach is based on a light illuminance sensor, which can calculate the distances between the target and lighting sources from the illuminance obtained by active control through a trilateration method. ${ }^{(18)}$ However, the problem of the effect of sunlight or human shadow must be solved for such localization. Alternatively, ultrasonic sensors are extensively used for localization since their measurement principle is simple to implement in hardware. ${ }^{(19,20)}$ In particular, the accuracy of ultrasonic sensors has reached the centimeter level. ${ }^{(21)}$ One of the disadvantages of ultrasonic sensors, however, is the detection angle limitation due to their radiation amplitude pattern. Interference among ultrasonic sensors also imposes another constraint when multiple sensors are used to localize objects in an environment.

As an attempt to address the above-mentioned problems caused by conventional ultrasonic sensors, in this paper, we propose a localization system based on omnidirectional ultrasonic sensing to circumvent the detection angle limitation of conventional ultrasonic sensors by using a reflection cone to generate ultrasonic beams with $360^{\circ}$ propagation. The architecture of the positioning system is composed of four ultrasonic receivers around a square measurement environment. The omnidirectional ultrasonic module is installed as a transmitter on a mobile robot. Each ultrasonic sensor is integrated with a Zigbee module for communication with a remote PC. ${ }^{(22,23)}$ When the ultrasonic sensor receivers receive an ultrasonic signal from the integrated omnidirectional ultrasonic module, distance measurements between the transmitter and the receiver are obtained by time-of-flight (TOF) calculation and sequentially transferred to the PC via the Zigbee wireless network. On the basis of the distance measurements collected from the receivers, the coordinates of the mobile robot are determined by using an established dual-circle localization method. ${ }^{(23)}$

The rest of the paper is organized as follows. Section 2 describes the omnidirectional ultrasonic module, which serves as a transmitter during the localization. The proposed dualcircle localization algorithm is derived in Sect. 3. Section 4 presents the architecture of the localization system based on omnidirectional ultrasonic sensing. Section 5 shows experimental results obtained when using the proposed localization system to estimate the position of a mobile robot in a measurement environment. Conclusions are drawn in Sect. 6. 


\section{Ultrasonic Sensors}

\subsection{Orientation limitation of ultrasonic sensors}

Ultrasound TOF sensors have been widely used in mobile robot applications such as distance measurement, environment perception, and robot navigation. However, they have been only marginally successful. In many applications such as environment perception or robot navigation, ultrasonic sensors have been considered unreliable and inaccurate, essentially owing to the inefficient use of the ultrasound information. ${ }^{(24)}$ Figure 1 shows the radiation amplitude pattern of a typical Polaroid 600 series transducer, ${ }^{(25)}$ where the beam is narrow and conical and has a number of secondary lobes representing the equipotential of the sound energy level. As a result, objects lying outside the detection geometry cannot be detected by the beam pattern because of the orientation limitation of the ultrasonic sensors.

\subsection{Proposed omnidirectional ultrasonic module}

The detection capability of ultrasonic sensors is significantly affected by their orientation limitation, therefore limiting the measurable area. In this paper, we integrate an ultrasonic sensor with a reflection cone mounted under an ultrasonic transmitter to reflect the incident ultrasonic signals from the ultrasonic transmitter to form an ultrasonic beam with $360^{\circ}$ radiation. Figure 2 shows a reflection cone made of Bakelite that we have designed as part of the omnidirectional ultrasonic module. The cone has a diameter of $2 \mathrm{~cm}$ and a height of $1 \mathrm{~cm}$ with an angle of inclination of $45^{\circ}$. Note that the diameter of the ultrasonic sensor of $1 \mathrm{~cm}$ is smaller than that of the reflection cone to ensure that the ultrasonic signals do not disperse. Figure 3 shows the integrated omnidirectional ultrasonic module as a transmitter comprising an ultrasonic sensor and a reflection cone. As illustrated in Fig. 3, the ultrasonic signals transmitted from the integrated omnidirectional ultrasonic module form a $360^{\circ}$ ultrasonic plane because of the reflection of the ultrasonic signals. ${ }^{(22,23)}$

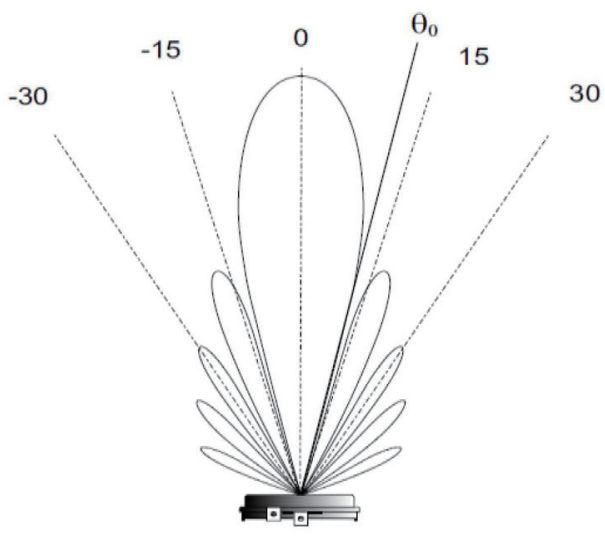

Fig. 1. Radiation pattern of a Polaroid 600 series transducer.

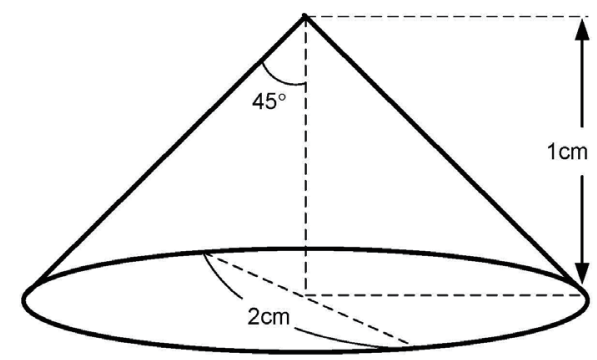

Fig. 2. Reflection cone designed as part of omnidirectional ultrasonic module. 


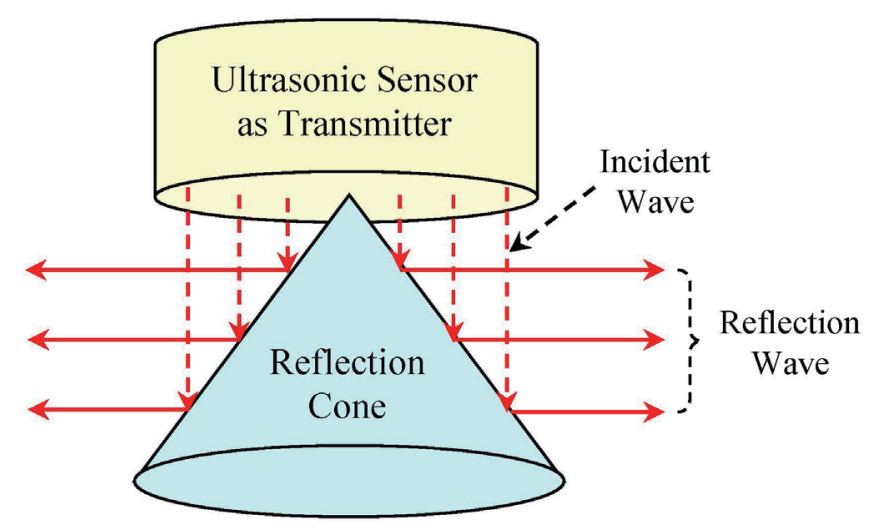

Fig. 3. (Color online) Integrated omnidirectional ultrasonic module.

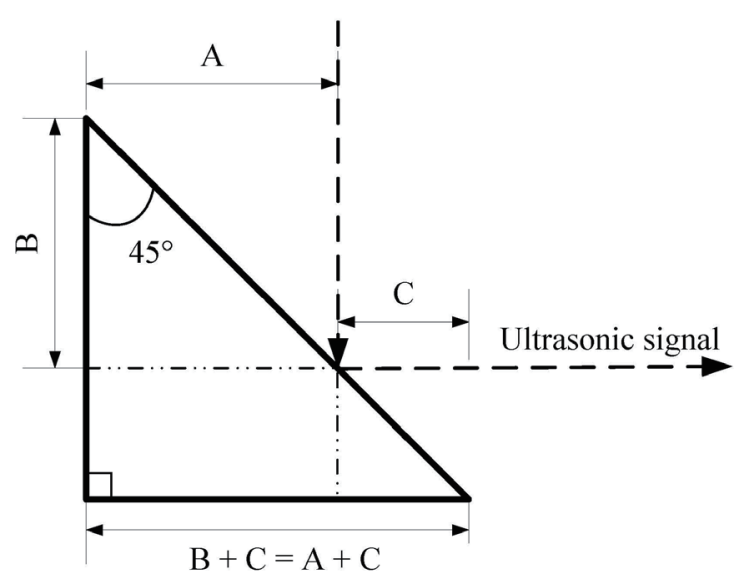

Fig. 4. Distance traveled by ultrasonic wave due to reflection.

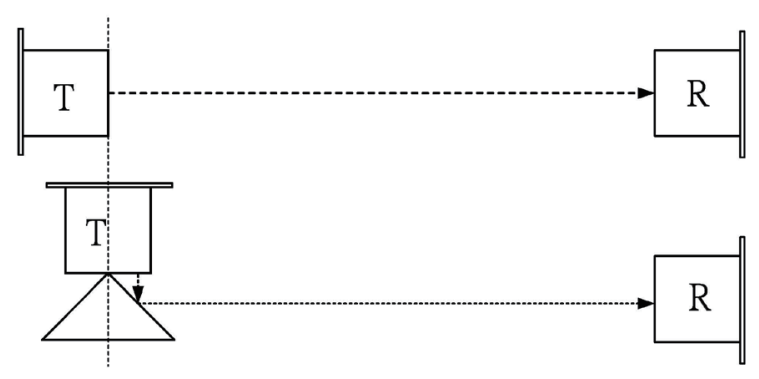

Fig. 5. Identical distance traveled by ultrasonic wave due to reflection.

Because of the geometric design of the reflection cone, the distance traveled by the ultrasonic wave due to reflection $(B+C)$ is the same from all directions, as if the ultrasonic signal is directly transmitted from the center of the cone without reflection (distance $A+C$ ), as illustrated in Fig. 4. ${ }^{(23)}$ As a result, the distance traveled by the ultrasonic wave due to the reflection cone is identical to the distance obtained when the system is deployed with the receiver directly opposite a transmitter, as demonstrated in Fig. 5.

As a result of the omnidirectional ultrasonic module, the ultrasonic pulses generated by the reflection cone propagate in the form of a $360^{\circ}$ ultrasonic plane as shown in Fig. 6, where ultrasonic receivers $U_{1}-U_{4}$ installed at different directions within a detectable area can easily receive the ultrasonic signal transmitted from a mobile robot $T$ equipped with an omnidirectional ultrasonic module. The omnidirectional ultrasonic module makes it unnecessary for the ultrasonic transmitter to repeatedly adjust its transmitting direction towards the ultrasonic receiver. 


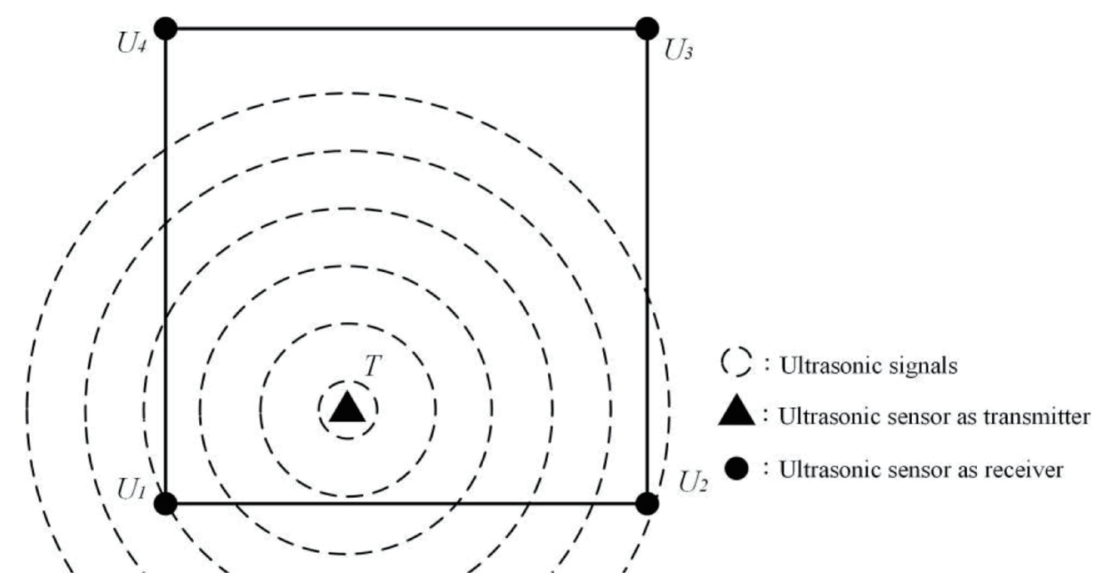

Fig. 6. Propagation of ultrasonic pulses generated by omnidirectional ultrasonic module.

\section{Dual-circle Localization Algorithm}

To determine the robot's position, a localization scheme is required. In this paper, we propose a dual-circle localization method ${ }^{(23)}$ to estimate the absolute position of a mobile robot with an omnidirectional ultrasonic module by collecting distances from its ultrasonic receivers. Figure 7 shows a simplified scheme to determine the coordinates of the robot by using the dual-circle localization method, where $U_{0}$ represents the omnidirectional ultrasonic module for transmitting the ultrasonic signal in all directions and $U_{1}-U_{4}$ are ultrasonic receivers for receiving ultrasonic signals from the omnidirectional ultrasonic module used to calculate the distances between the robot and the receivers. Assume that the coordinates of $U_{0}, U_{1}, U_{2}, U_{3}$, and $U_{4}$ are $(X, Y),\left(X_{1}, Y_{1}\right)$, $\left(X_{2}, Y_{2}\right),\left(X_{3}, Y_{3}\right)$, and $\left(X_{4}, Y_{4}\right)$ and that the distances between $U_{1}$ and $U_{0}, U_{2}$ and $U_{0}$, and $U_{3}$ and $U_{0}$, are $R_{1}, R_{2}$, and $R_{3}$, respectively. The objective of the dual-circle localization method is to determine the coordinates $U_{0}(X, Y)$ given the distance measurements of $R_{1}, R_{2}$, and $R_{3}$.

According to basic circular geometry, the intersection of the circle centered at $U_{1}$ with radius $R_{1}$ and the circle centered at $U_{2}$ with radius $R_{2}$ can be obtained by solving the equations

$$
\begin{aligned}
& \left(X-X_{1}\right)^{2}+\left(Y-Y_{1}\right)^{2}=R_{1}{ }^{2}, \\
& \left(X-X_{2}\right)^{2}+\left(Y-Y_{2}\right)^{2}=R_{2}^{2} .
\end{aligned}
$$

If $Y_{1}=Y_{2}$, we obtain

$$
\left(X-X_{1}\right)^{2}-\left(X-X_{2}\right)^{2}=R_{1}^{2}-R_{2}^{2},
$$

by subtracting Eq. (2) from Eq. (1). Therefore, the intersection point $(X, Y)$ can be obtained as 


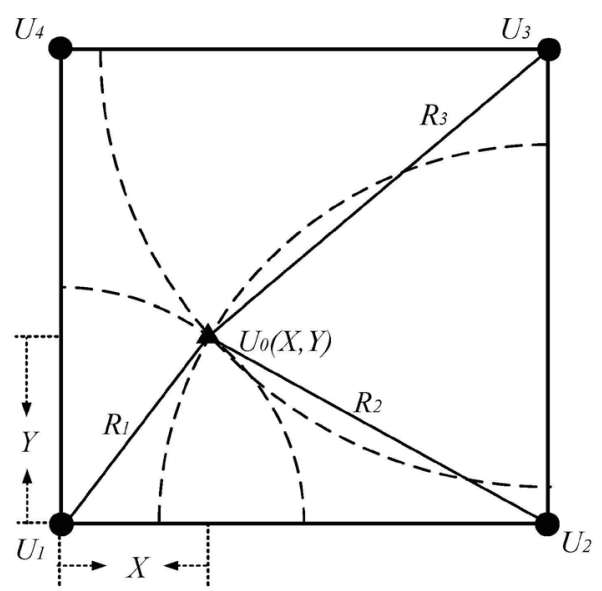

Fig. 7. Simplified scheme of dual-circle localization method.

$$
\begin{gathered}
X=\frac{R_{1}^{2}-R_{2}^{2}-X_{1}^{2}+X_{2}^{2}}{2\left(X_{2}-X_{1}\right)}, \\
Y=\sqrt{R_{1}^{2}-X^{2}+2 X \cdot X_{1}-X_{1}^{2}-Y_{1}^{2}+2 Y \cdot Y_{1}} .
\end{gathered}
$$

Consider the case where $Y_{1} \neq Y_{2}$. The intersection of the circle centered at $U_{2}$ with radius $R_{2}$ and the circle centered at $U_{3}$ with radius $R_{3}$ can be obtained by solving the equations

$$
\begin{aligned}
& \left(X-X_{2}\right)^{2}+\left(Y-Y_{2}\right)^{2}=R_{2}{ }^{2}, \\
& \left(X-X_{3}\right)^{2}+\left(Y-Y_{3}\right)^{2}=R_{3}{ }^{2} .
\end{aligned}
$$

Subtracting Eq. (7) from Eq. (6), we obtain

$$
2\left(X_{3}-X_{2}\right) X+2 Y\left(Y_{3}-Y_{2}\right)=R_{2}{ }^{2}-R_{3}{ }^{2}+X_{3}{ }^{2}-X_{2}{ }^{2}+Y_{3}{ }^{2}-Y_{2}{ }^{2} .
$$

After rearrangement, $Y$ is given by

$$
Y=M X+K,
$$

where $M$ and $K$ are expressed as

$$
M=\frac{X_{2}-X_{3}}{Y_{3}-Y_{2}}
$$




$$
K=\frac{R_{2}^{2}-R_{3}^{2}+X_{3}^{2}-X_{2}^{2}+Y_{3}^{2}-Y_{2}^{2}}{2\left(Y_{3}-Y_{2}\right)} .
$$

Substituting Eq. (9) into Eq. (7), we obtain

$$
\left(X-X_{3}\right)^{2}+\left(M \cdot X+K-Y_{3}^{2}\right)=R_{3}{ }^{2},
$$

which can be simplified as

$$
A \cdot X^{2}+B \cdot X+C=0,
$$

where $A, B$, and $C$ are expressed as

$$
\begin{gathered}
A=1+M^{2}, \\
B=2\left(M \cdot K-M \cdot Y_{3}-X_{3}\right), \\
C=X_{3}{ }^{2}+Y_{3}{ }^{2}+K^{2}-2 K \cdot Y_{3}-R_{3}{ }^{2} .
\end{gathered}
$$

Finally, $X$ can be obtained as

$$
X=\frac{-B \pm \sqrt{B^{2}-4 A \cdot C}}{2 A}
$$

by solving Eqs. (13)-(16) and $Y$ can be found from Eq. (9). Thus, we have obtained the coordinates $U_{0}(X, Y)$ of the mobile robot. Note that only two ultrasonic receivers are sufficient to determine the coordinates of the robot. Using three or four ultrasonic receivers, however, will help enhance the precision of measurement through sensor data fusion techniques. ${ }^{(26)}$

\section{Omnidirectional Ultrasonic Sensing Localization}

To estimate the position of a target object, such as the mobile robot, distance measurements must be obtained via the ultrasonic sensors before we can calculate the coordinates of the robot by using the dual-circle localization method described in Sect. 3. Because of communication requirements, Zigbee wireless modules are incorporated into the omnidirectional ultrasonic module and ultrasonic receivers to receive commands from a remote PC and transmit distance measurements to the remote PC to calculate the position of the mobile robot. 


\subsection{Localization scheme}

Figure 8 shows the localization scheme based on omnidirectional ultrasonic sensing in an environment where the target is a mobile robot carrying an integrated omnidirectional ultrasonic module $U_{0}$ as a transmitter with Zigbee wireless communication capability. ${ }^{(22,23)}$ A microcontroller embedded with the Zigbee module is required to activate the ultrasonic sensors. Four ultrasonic receivers with Zigbee wireless communication capability are positioned around the measuring site, forming a square in the experimental environment. Because the Zigbee modules are integrated with ultrasonic sensors, a wireless network can be established for seamless communication between the ultrasonic sensors and the remote PC. As a result, the PC can directly give instructions to the integrated omnidirectional ultrasonic module (transmitter) and the four ultrasonic receivers over the wireless communication network. Distance measurements collected by the four receivers are then transferred to the PC for processing to estimate the position of the robot by using the established dual-circle localization method. By using the proposed method based on omnidirectional ultrasonic sensing, the angle limitation constraint imposed on conventional ultrasonic sensors is therefore overcome.

\subsection{Hardware configuration}

Figure 9 shows the hardware configuration of the proposed localization scheme based on omnidirectional ultrasonic sensing. As the first step, the remote PC issues a receiving command to all of the ultrasonic receivers over the Zigbee wireless network to instruct them to get ready to receive ultrasonic signals. Immediately after issuing a receiving command, the remote PC then issues a transmission command to the integrated omnidirectional ultrasonic module over the

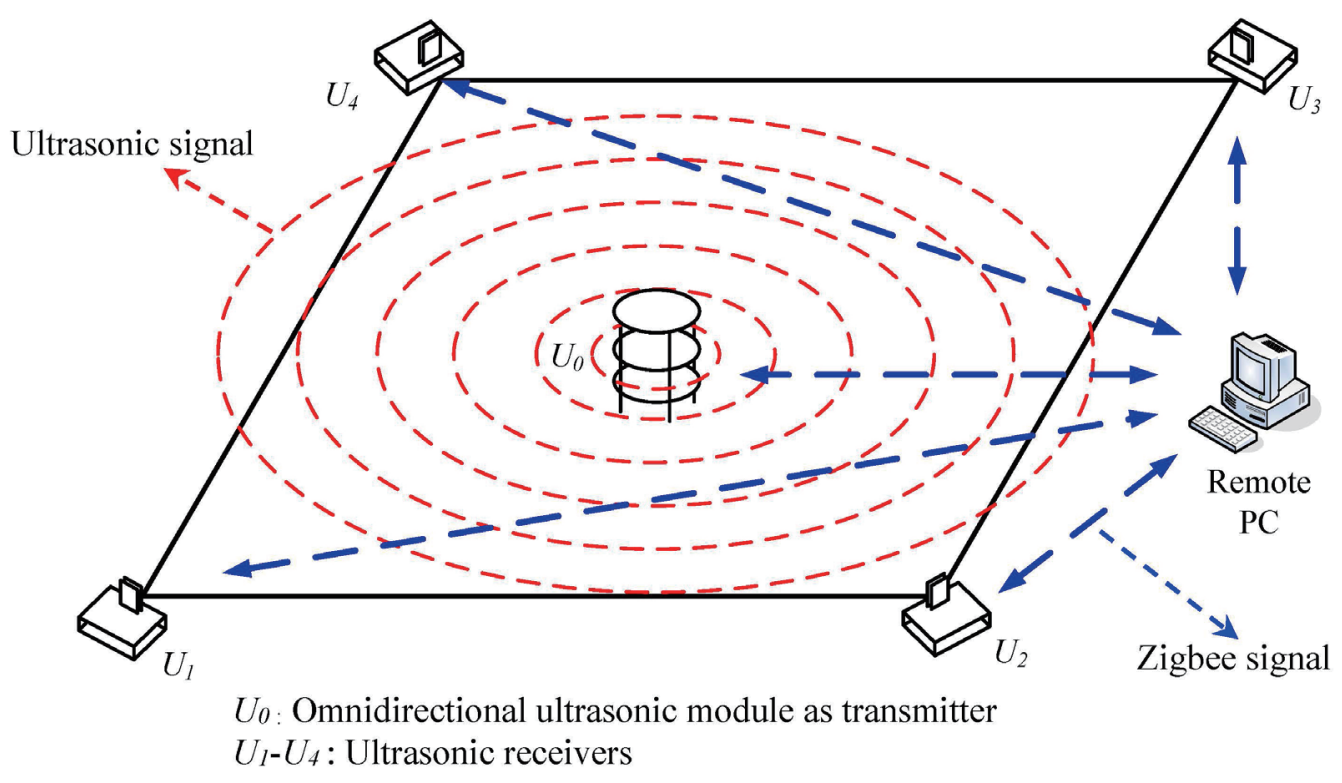

Fig. 8. (Color online) Localization scheme based on omnidirectional ultrasonic sensing. 


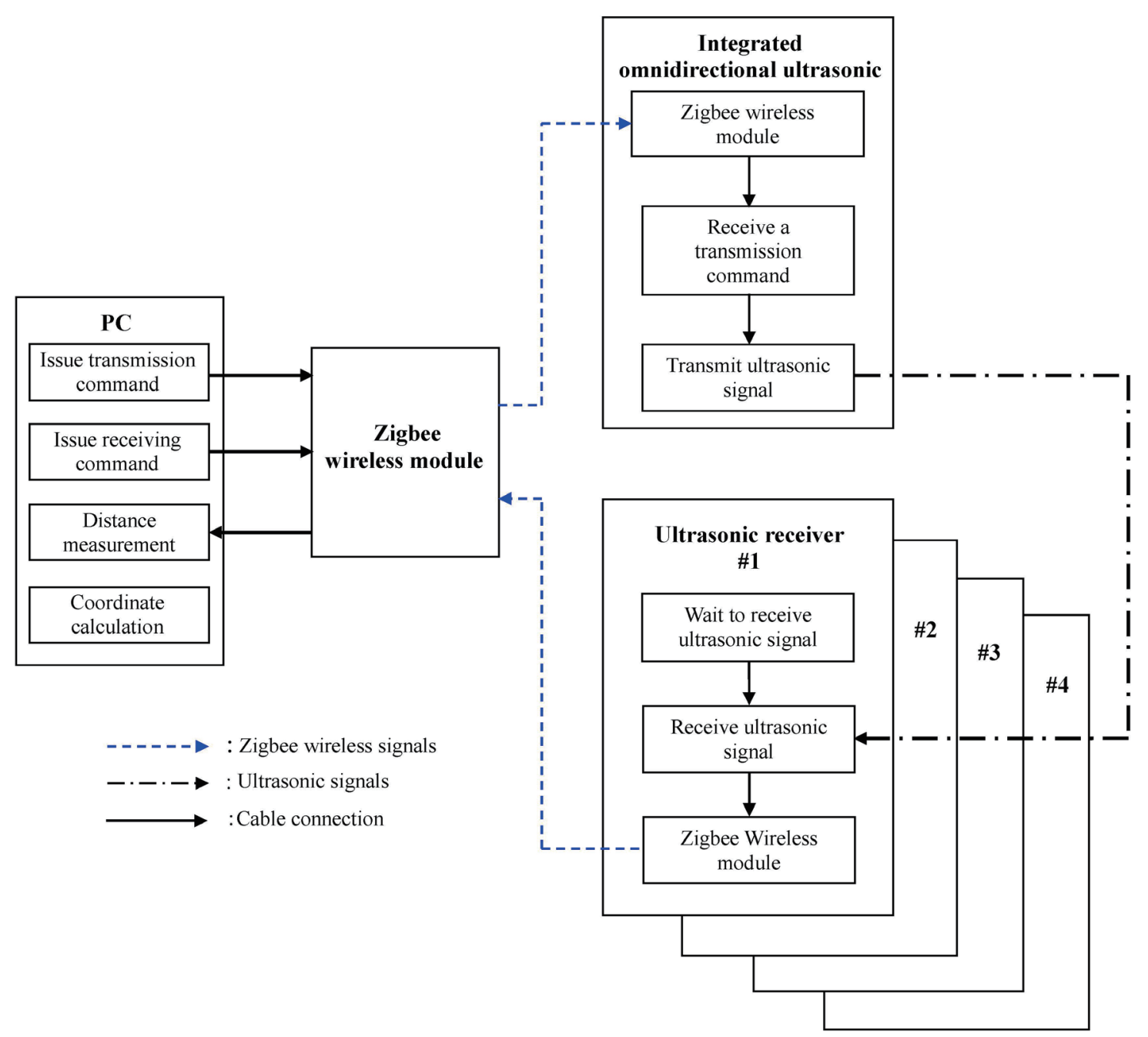

Fig. 9. (Color online) Hardware configuration of omnidirectional ultrasonic sensing localization scheme.

Zigbee wireless network to transmit ultrasonic signals towards the ultrasonic receivers. When the ultrasonic receivers receive the ultrasonic signals from the integrated omnidirectional ultrasonic module, distance measurements between the transmitter and the receiver can be obtained by TOF calculation, which are sequentially transferred to the PC via the Zigbee wireless network. Using the distance measurements collected from the receivers, the position of the mobile robot can be determined by the dual-circle localization method.

To establish a wireless communication network, Zigbee modules are incorporated into the PC, the integrated omnidirectional ultrasonic module, and the ultrasonic receivers. The Zigbee module we adopted in this paper, XBee, provides three communication modes: peer-to-peer, waiting, and broadcasting. In this paper, the broadcasting mode is used because each receiver passively receives commands from the remote PC, which is suitable for the broadcasting mode. Its simplicity in terms of the configuration and implementation is also an advantage. 


\subsection{Localization procedures}

Figure 10 shows a flowchart of the proposed localization system based on omnidirectional ultrasonic sensing. ${ }^{(21-23)}$ Initially, the PC issues a command via the Zigbee wireless network to instruct all four ultrasonic receivers to prepare to receive signals. At the same time, a command is issued to the ultrasonic transmitter on the robot over the Zigbee wireless network to transmit ultrasonic signals towards the receivers. The ultrasonic signals received by the receivers that are converted into distance measurements using the TOF all have the same frequency of $40 \mathrm{kHz}$. Without an identification procedure, interference is inevitably encountered when all four receivers receive signals at the same time. To identify the corresponding relationship between the distance measurements and the receivers, a sequential polling operation is used to control the ultrasonic receivers so that they transfer their distance measurements one by one. Figure 11

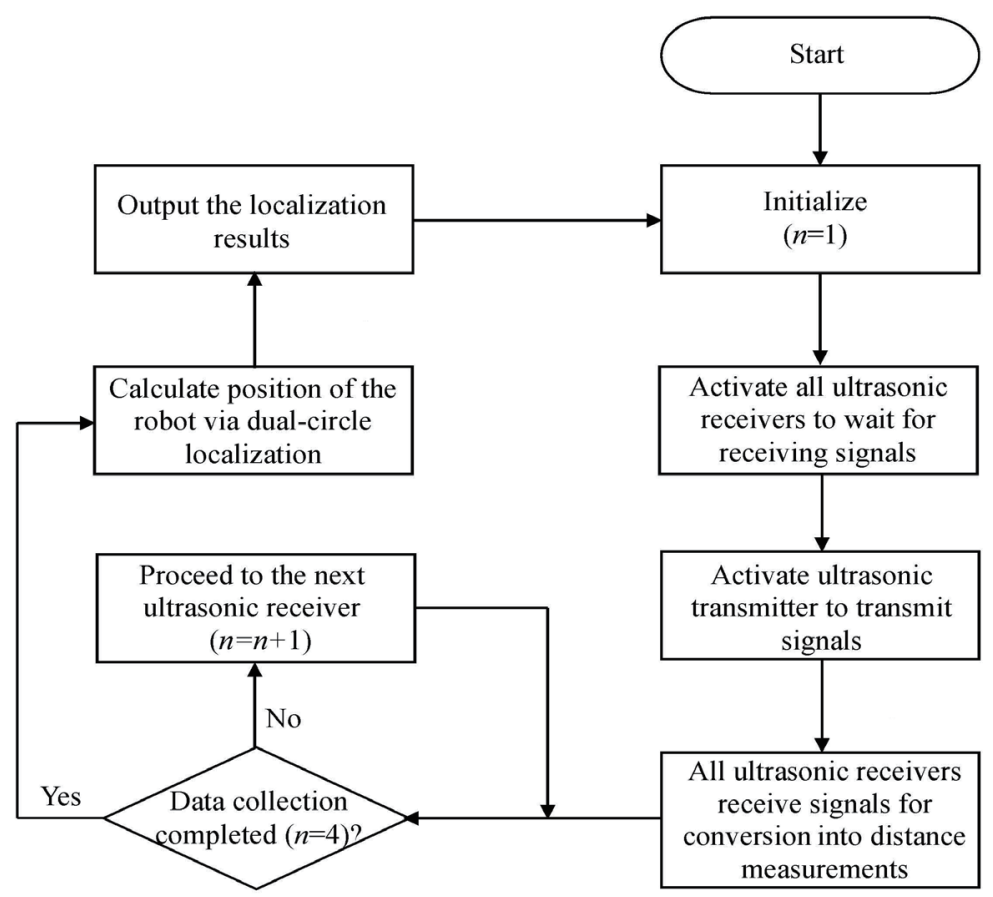

Fig. 10. Flowchart of proposed omnidirectional ultrasonic sensing localization system.

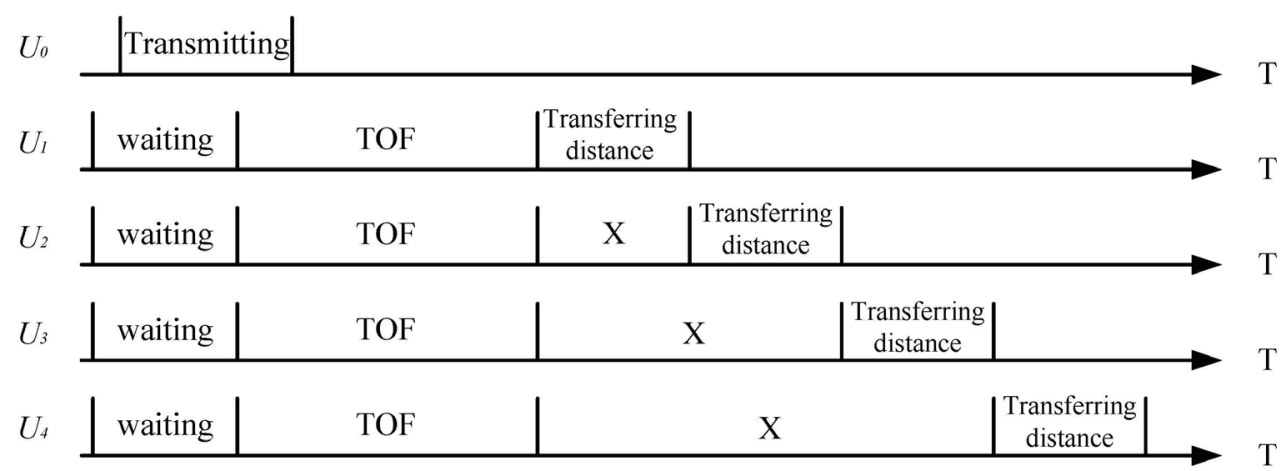

Fig. 11. Communication timing of ultrasonic sensors for distance measurement based on polling scheme. 
shows a timing chart illustrating the sequences of the ultrasonic sensors transmitting/receiving ultrasonic signals to obtain distance measurements based on a polling scheme. After the polling cycle is completed when the PC has collected all the distance measurements from the receivers, the position of the robot can then be calculated by the dual-circle localization method.

\section{Experimental Results}

In our experiments, we use Devantech SFR02 ultrasonic sensors having an opening angle of around $\pm 30^{\circ}$ and $\mathrm{XBee}$ Zigbee modules to construct ultrasonic transmitter and receiver modules with wireless communication capability. Each ultrasonic sensor has a unique ID number for easy identification. Figures 12 and 13 show the integrated omnidirectional ultrasonic module incorporating a reflection cone and a Zigbee module as the transmitter and the ultrasonic module incorporating a Zigbee module as the receiver, respectively. The measuring site is a square of side $4 \mathrm{~m}$, where the measuring area is confined to an area of $3 \mathrm{~m}$ by $3 \mathrm{~m}$. There are nine locations uniformly sampled for testing by driving the mobile robot in the measuring environment as shown in Fig. 14. ${ }^{(23)}$ Since the sound speed is $340 \mathrm{~m} / \mathrm{s}$ and the farthest receiving distance in the 3

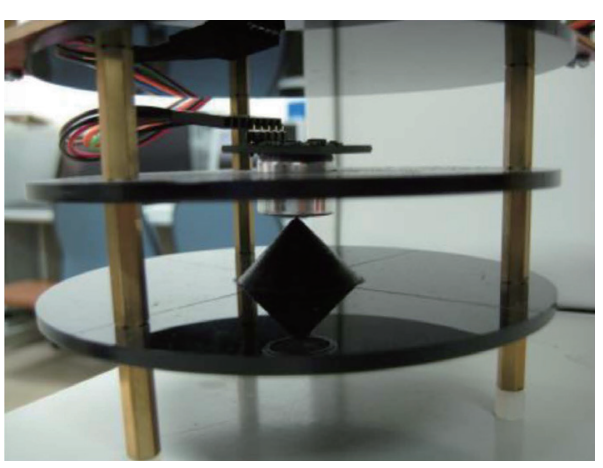

Fig. 12. (Color online) Integrated omnidirectional ultrasonic module incorporating Zigbee module as transmitter.

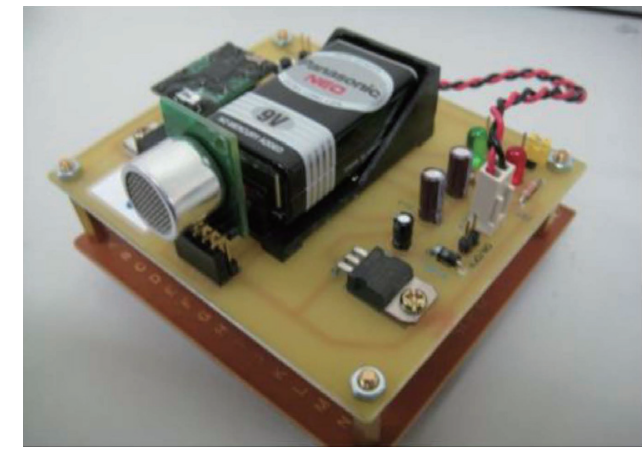

Fig. 13. (Color online) Ultrasonic module incorporating Zigbee module as receiver.

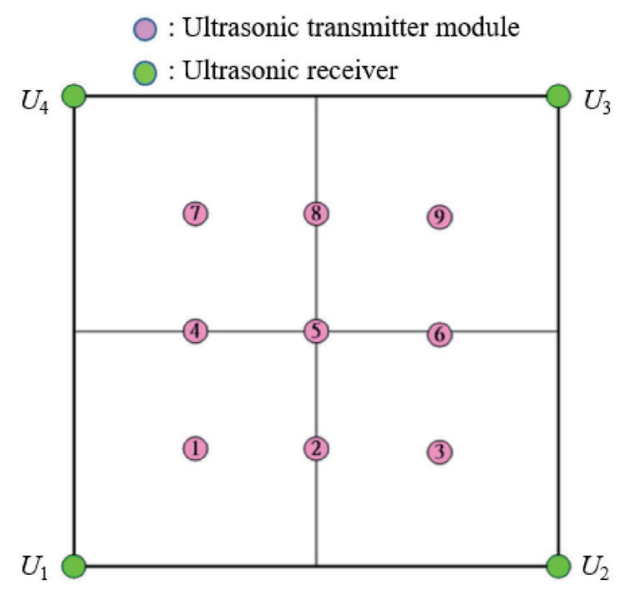

Fig. 14. (Color online) Test points for localization in the measuring environment. 
m by $3 \mathrm{~m}$ measuring area is $4.24 \mathrm{~m}$, the TOF required for the transmitter/receiver ultrasonic modules from transmitting to receiving is only $12.5 \mathrm{~ms}$ (4.24/340). In addition, the transmission rate of the Zigbee module is $250 \mathrm{kbps}$ at a frequency of $2.4 \mathrm{GHz}$, and thus the transmission time of the Zigbee module is only $32 \mathrm{~ms}$ if $1 \mathrm{kB}$ data is transmitted.

Experimental results obtained using the proposed localization method based on omnidirectional ultrasonic sensing are listed in Table 1. ${ }^{(23)}$ All measurement errors lie within an acceptable range of $\pm 6 \mathrm{~cm}$, and the average absolute error in the coordinates is approximately $(2.5,3.22)$. In addition, we change the localization algorithm to triangulation localization and observe the measurement results, as shown in Table 2. ${ }^{(22)}$ We find that the average absolute error in the coordinates is about $(2.89,3.11)$; therefore, the proposed omnidirectional ultrasonic localization has good localization accuracy regardless of which of the two localization methods is used. Note that distance measurements are collected by sequentially polling the receivers transferring their measurements to avoid possible interference. As a result, each receiver is activated in sequence to transfer its distance measurement one at a time. The data collection efficiency is slightly reduced by the polling process. However, for the navigation of the mobile robot, whose coordinates can be instantly determined, the impact is minor and can therefore be neglected.

Two well-known technologies, WiFi localization and Bluetooth localization, rely on the received signal strength indicator (RSSI) to determine the position, and their localization accuracies are $10-15 \mathrm{~m}$ and $1-3 \mathrm{~m},{ }^{(27)}$ respectively. According to the present experimental

Table 1

Experimental results of proposed system using dual-circle localization.

\begin{tabular}{lccc}
\hline Point number & Actual coordinates $(\mathrm{cm})$ & Measured coordinates $(\mathrm{cm})$ & Measurement error $(\mathrm{cm})$ \\
\hline 1 & $(100,100)$ & $(104,99)$ & $(4,-1)$ \\
2 & $(200,100)$ & $(200,101)$ & $(0,1)$ \\
3 & $(300,100)$ & $(302,105)$ & $(2,5)$ \\
4 & $(100,200)$ & $(104,198)$ & $(4,-2)$ \\
5 & $(200,200)$ & $(204,197)$ & $(4,-3)$ \\
6 & $(300,200)$ & $(300.5,198.5)$ & $(0.5,-1.5)$ \\
7 & $(100,300)$ & $(104.5,295)$ & $(4.5,-5)$ \\
8 & $(200,300)$ & $(202.5,294)$ & $(2.5,-6)$ \\
9 & $(300,300)$ & $(301,295.5)$ & $(1,-4.5)$ \\
\hline
\end{tabular}

Table 2

Experimental results of proposed system using triangulation localization.

\begin{tabular}{lccc}
\hline Point number & Actual coordinates $(\mathrm{cm})$ & Measured coordinates $(\mathrm{cm})$ & Measurement error $(\mathrm{cm})$ \\
\hline 1 & $(100,100)$ & $(101,99)$ & $(1,-1)$ \\
2 & $(200,100)$ & $(196,101)$ & $(-4,1)$ \\
3 & $(300,100)$ & $(295,103)$ & $(-5,3)$ \\
4 & $(100,200)$ & $(100,198)$ & $(0,-2)$ \\
5 & $(200,200)$ & $(198,197)$ & $(-2,-3)$ \\
6 & $(300,200)$ & $(293,198)$ & $(-7,-2)$ \\
7 & $(100,300)$ & $(101,296)$ & $(1,-4)$ \\
8 & $(200,300)$ & $(198,294)$ & $(-2,-6)$ \\
9 & $(300,300)$ & $(296,294)$ & $(-4,-6)$ \\
\hline
\end{tabular}


results, the proposed localization based on omnidirectional ultrasonic sensing can achieve an accuracy of less than $10 \mathrm{~cm}$. Therefore, the proposed localization method has superior localization accuracy to the other methods using RSSI detection. Using the reflection cone integrated with an ultrasonic sensor as an ultrasonic transmitter overcomes the disadvantages of the detection angle limitation due to the radiation amplitude pattern of the ultrasonic sensor. However, the interference among ultrasonic sensors also becomes a constraint when multiple sensors are used for localization.

\section{Conclusions}

In this paper, we presented a novel localization system for mobile robots based on omnidirectional ultrasonic sensing that overcomes the angle limitation of conventional ultrasonic sensors by using a reflection cone to generate ultrasonic waves with $360^{\circ}$ propagation. To provide seamless communication while avoiding interference in the measuring environment, Zigbee modules are integrated into all ultrasonic sensors to transmit commands and receive measurements by sequential control. Because of the use of a dual-circle localization scheme, the coordinates of a mobile robot carrying the integrated omnidirectional ultrasonic transmitter can be determined using the distance measurements obtained by the ultrasonic receivers deployed in the measuring environment. Experimental results have shown a satisfactory accuracy of $\pm 6 \mathrm{~cm}$ in measuring the coordinates of the mobile robot by the proposed localization system. Because as many as six sets of distance measurements can be obtained at one time to determine the position of the robot, sensor fusion techniques, for example, the Dempster-Shafer technique or fuzzy logic, can be incorporated to obtain more reliable measurements in the future.

\section{Acknowledgments}

This work was partially supported by the National Science Council, Taiwan, under Grant 100-2221-E-003-005-MY2.

\section{References}

1 M. Piasecki: Rob. Auton. Syst. 16 (1995) 93. https://doi.org/10.1016/0921-8890(95)00037-G

2 D. Tan, Q. Wang, Y. Wang, and R. Cao: Mach. Electron. 9 (2005) 46. https://doi.org/10.1109/RME.2005.1542933

3 J. Tang, T. Bao, and Z. Cai: Control Autom. 5 (2005) 20. https://doi.org/10.1109/ICCAS.2007.4406753

4 P. Enge and P. Misra: Proc. IEEE 87 (1999) 3. https://doi.org/10.1109/JPROC.1999.736338

5 C. Zong, Z. Ji, Y. Yu, and H. Shi: Sens. Mater. 32 (2020) 1159. https://doi.org/10.18494/SAM.2020.2540

6 S. A. Li, L. H. Chou, T. H. Chang, C. H. Yang, and Y. C. Chang: Sens. Mater. 31 (2020) 1021. https://doi. org/10.18494/SAM.2019.2226

7 Y. Li, D. Xu, F. Li, M. Bai, and S. Zhang: Trans. Chin. Soc. Agric. Eng. 1 (2005) 66. https://www.researchgate. net/publication/295755270_Application_of_GPS technology_in_agricultural land levelling_survey

8 S. Chen: J. Wuhan Univ. Technol. 4 (2002) 448. https://en.cnki.com.cn/Ärticle en/CJFDTOTALJTKJ200204007.htm

9 Z. B. Zhang, S. H. Ye, Z. Q. Xu, Y. H. Wang, and Y. Z. Zheng: ACTA Metrologica Sinica 2 (2002) 98. https:// en.cnki.com.cn/Article_en/CJFDTOTAL-JLXB200202004.htm

10 S. Corbellini, F. Ferraris, and M. Parvis: IEEE Trans. Instrum. Meas. 55 (2006) 1530. https://doi.org/10.1109/ TIM.2006.880266 
11 H. Temeltas and D. Kayak: IEEE Aerosp. Electron. Syst. Mag. 23 (2008) 16. https://doi.org/10.1109/ MAES.2008.4694832

12 E. Menegatti, A. Pretto, A. Scarpa, and E. Pagello: IEEE Trans. Robot. 22 (2006) 523. https://doi.org/10.1109/ TRO.2006.875495

13 C. C. Hsu, C. C. Wong, H. C. Teng, N. J. Li, and C. Y. Ho: Int. J. Innov. Comput. Inf. Control. (IJICIC) 7 (2011) 3725. https://doi.org/10.1109/ICICIC.2006.327

14 J. S. Chiang, C. H. Hsia, and H. W. Hsu: IEEE Sens. J. 13 (2013) 1677. https://doi.org/10.1109/ JSEN.2013.2240449

15 A. F. C. Errington, B. L. F. Daku, and A. F. Prugger: IEEE Trans. Instrum. Meas. 59 (2010) 2863. https://doi. org/10.1109/TIM.2010.2046366

16 J. Ke, H. Shen, and X. Wei: Mod. Electron. Tech. 23 (2006) 12. https://en.cnki.com.cn/Article_en/ CJFDTOTAL-XDDJ200623004.htm

17 M. H. Zhang, S. S. Zhang, and J. Cao: Comput. Sci. 24 (2007) 68. https://en.cnki.com.cn/Article_en/ CJFDTOTAL-JSJA200706018.htm

18 K. Moriya, M. Fujimoto, Y. Arakawa, and K. Yasumoto: Sens. Mater. 32 (2020) 625. https://doi.org/10.18494/ SAM.2020.2613

19 Y. L. Zhu: Dev. Inno. Mach. Electr. Products 19 (2006) 128. https://en.cnki.com.cn/Article_en/CJFDTOTALJDCP200602050.htm

20 K. Zhang and G. Liu: Mod. Electron. Tech. 15 (2007) 139. https://api.semanticscholar.org/CorpusID:113565827

21 C. C. Hsu, C. Y. Lai, J. W. Huang, and Y. H. Chen: J. Harbin Inst. Tech. 15 (2008) 185. http://tkuir.lib.tku.edu. tw:8080/dspace/handle/987654321/42048

22 C. C. Hsu, H. C. Chen, and C. Y. Lai: Proc. 2009 Int. Asia Conf. Informatics in Control, Automation and Robotics (CAR, 2009) 437. https://doi.org/10.1109/CAR.2009.93

23 C. C. Hsu, C. Y. Lai, C. Kanamori, H. Aoyama, and C. C. Wong: Proc. 2011 SICE Annual Conf. (SICE, 2011) 1972. https://ieeexplore.ieee.org/stamp/stamp.jsp?tp=\&arnumber $=6060291$

24 F. Moita, A. C. Lopes1, and U. Nunes: J. Intell. Robot. Syst. 50 (2007) 141. https://doi.org/10.1007/s10846-0079158-5

25 D. MacIsaac and A. Hämäläinen: Phys. Teach. 40 (2002) 39. https://doi.org/10.1119/1.1457828

26 W. Koch: IEEE Aerosp. Electron. Syst. Mag. 25 (2010) 29. https://doi.org/10.1109/MAES.2010.5546307

27 WiFi or Bluetooth Beacons for Indoor Location: https://www.pointr.tech/blog/wifi-or-beacons-for-indoorlocation (accessed July 2021). 